\title{
ASSESSMENT OF LEVEL-OF-SERVICE ON URBAN ARTERIALS: A CASE STUDY IN KOLKATA METROPOLIS
}

\author{
Subhadip Biswas ${ }^{1}$, Bhupendra Singh ${ }^{2}$, Arpita Saha ${ }^{3}$ \\ 1, 2, 3 Civil Engineering Department, Indian Institute of Technology Roorkee, Roorkee-247667, \\ Uttarakhand, India
}

Received 18 June 2016; accepted 15 August 2016

\begin{abstract}
Mixed traffic conditions in emerging countries like India make it difficult to adopt Level of Service (LOS) criteria given in Highway Capacity Manual (HCM) of developed nations. Present study aims at identifying alternative performance measure which will exhibit better compatibility to define LOS in context of urban mixed traffic. A total sixteen hours of traffic volume and speed data have been recorded by videography at selected road segment of a six lane divided urban arterial in Kolkata metropolis. Percentage Speed Reduction (PSR) from Free Flow Speed (FFS) has been identified as an alternative performance measure for LOS assessment as it is a good representative of overall mobility state and also found sensitive to prevailing traffic flow conditions on the road. FFS of individual vehicle category has been computed from the normal distribution curves fitted upon the speed data under free flowing condition. Kolmogorov-Smirnov (K-S) test which has been performed to check goodnessof-fit of these modelled curves, has shown satisfactory compatibility with the observed data. K-mean clustering has been adopted to classify the observed PSR data into sub groups and consequently Silhouette method has been used to validate these clusters. Finally, six LOS classes bounded by threshold values of PSR have been proposed.
\end{abstract}

Keywords: arterial, level of service, performance measure, free flow speed, clustering.

\section{Introduction}

Since independence, India has witnessed tremendous economic growth that resulted rapid urbanization and growing needs of transportation as a subsequence (Lal and Clement, 2005; Singh, 2014). The existing infrastructures thus became quite inadequate to cater the needs and also do not warrant to provide the desired service level. At the same time, the mixed traffic composed of a wide range of vehicle categories made it difficult to select appropriate lane configuration as capacity analysis and assessment of level-of- service (LOS) for such mixed traffic is quite complicated. Also, recommended LOS criteria in Highway Capacity Manuals of developed nations may not be adoptable in context of India or other developing countries due to difference in traffic characteristics and driving culture. Besides, the ribbon development, encroachment and many others affect the service level provided to the users as well. The present study thus, aimed at identifying suitable alternative performance measure and developing a method which would exhibit compatibility with prevalent heterogeneous traffic for the determination of LOS.

\footnotetext{
${ }^{1}$ Corresponding author: subha.dce2014@iitr.ac.in
} 
Highway Capacity Manual (HCM) (1965) first introduced the concept of LOS and some corresponding performance measures which significantly represent the operating characteristics of a roadway. Six LOS were suggested based on some performance measures like average travel speed, peak hour factor, v/c ratio, load-factor at intersection and flow condition (stable, unstable or forced). In HCM (1985), density was selected as the primary measure for performance assessment and correspondingly five LOS were proposed. HCM (2000) suggested average travel speed (ATS) as the exclusive parameter for assessment of LOS of urban street. Six LOS criteria were proposed on the basis of ATS value for four urban street classes individually whereas urban street class was determined based on the free flow speed. In most recent edition HCM (2010), six LOS were suggested along with threshold values of percent free-flow speed which was introduced as the main performance measure for LOS assessment of urban street for the automobile mode. However, according to many research outcomes, LOS criteria suggested in HCM may not perfectly fit for urban roads under prevailing mixed traffic condition. For example, Bhuyan and Rao (2011) and Das et al. (2013) defined LOS criteria for Indian mixed traffic condition based on ATS, the performance measure suggested in HCM (2000) and found threshold values of ATS significantly different from those proposed in HCM. Apart from conventional performance measures, few researchers recognized some alternative parameters that would be more appropriate in characterizing serviceability of a mixed traffic stream. Maitra et al. (1999) considered 'congestion' as a sole parameter to define LOS and consequently postulated a model to quantify the level of congestion. Ten LOS classes were therefore proposed based on different congestion levels. In reviewing literature based on congestion measurement, Rao and Rao (2012) also put forward a few congestion based approaches for LOS evaluation. On the other hand, Marwah and Singh (2000) realized LOS of urban streets related to multiple parameters instead of a single factor. Journey speed, concentration and road occupancy were hence, considered as explicit operating characteristics and on the basis of that, four distinct classes of level of service were suggested. However, disadvantage of both these approaches (Maitra et al., 1999; Marwah and Singh, 2000) lies in its difficulties to estimate performance measures specifically congestion or concentration. Also in evaluating LOS thresholds, no state-of-theart classification technique was adopted in either of these studies. Thus, all these had motivated the present study to identify an alternative performance measure and assess LOS in context of urban mixed traffic.

\section{Percent Speed Reduction: An Identified Performance Measure}

Speed which directly reflects the mobility condition of a section, is well representative of the serviceability of an urban arterial. However, speed at mid-block section is dependent on traffic circumstance as well as roadway condition also. Therefore, speed as a sole performance measure is only transferable to other roadway segment having similar road geometry. Alternatively, free flow speed (FFS) which is a constant value for a particular roadway segment, well indicates the prevailing roadway characteristics by mobility approach. Now, percentage speed reduction (PSR) from FFS may be taken as a performance measure as it is transferable to any other urban road segment. For example, quality of services experienced by two 
vehicles travelling at different speeds with same PSR value on different road segments, are pretended to be same. HCM (2010) also suggested Percentage of Free Flow Speed as a performance measure for LOS. But in estimation of FFS, categorization of vehicles was not taken into consideration. On the other hand, in context of urban mixed traffic conditions of developing countries, FFS should be considered vehicle category wise as different types of vehicle having different static and dynamic characteristics, would have different FFS values. Therefore as per requirement, FFS study was essential to determine FFS separately for different vehicle categories under prevailing roadway condition.

\section{Field Data Collection and Free Flow Speed Study}

Field studies were carried out adopting videography technique to observe the traffic flow characteristics on urban arterials in Kolkata metropolis. A straight road segment of $60 \mathrm{~m}$ length was selected on E.M.Bypass, a six lane divided urban arterial with $10 \mathrm{~m}$ single directional width. Segment was free from street parking, bus stop and pedestrian activities and also it was far from any intersection. To capture the movement of vehicles at a wide range of volume levels, camera was kept on a tripod stand placing it on terrace of a $\mathrm{G}+3$ building which is $30 \mathrm{~m}$ away from the nearest edge of carriageway. Camera had been adjusted in such a way that entire segment of $60 \mathrm{~m}$ span could be captured without trouble. A total 16 hours (6 am to $10 \mathrm{pm}$ ) of video data were collected during which weather was clear and no precipitation occurred. Later, required traffic speed and volume data were extracted by playing the video files on a computer. As per the observation, vehicles were classified into six categories: motorized two wheeler, small car, big car, bus, Light Commercial Vehicle (LCV) and truck. New generation larger sized cars (like SUV) featured with more than 2500 $\mathrm{cc}$ engine were taken into big car category and the rest of all cars were considered as small car. Entry and exit time of individual vehicle on the previously marked $60 \mathrm{~m}$ long trap were recorded with accuracy of 0.01 second. Corresponding speed was measured from the duration of time spent by the vehicle to cross the trap.

Free flow condition generally appears in low traffic flow situation where movement of a vehicle would not be affected by the presence of other vehicles in traffic stream. The speed corresponding to such flow level is termed as free flow speed (FFS). HCM (2010) considers a vehicle free flowing if it has headway of 8 second and more to the preceding vehicle and 5 second or more to the following vehicle. Present study adopts this notion for field measurement of FFS. Vehicles satisfying the free flowing criteria were considered separately and mean of those corresponding speed values were taken as FFS. In a mixed traffic situation, different types of vehicles are expected to move at different speeds even at low traffic flow level. Thus, category wise speed data were collected at free-flow condition and fitted to normal distribution in order to assess the mean. Probability Density Function (PDF) of normal distribution is presented in Eq. (1).

$\operatorname{PDF}(x)=\frac{1}{\sigma \sqrt{2} \pi} e^{-\frac{(x-\mu)^{2}}{2 \sigma^{2}}}$

Where, $\mu$ is the mean and $\sigma$ is the standard deviation of the variable $x$. 
As expected, Free Flow Speeds of different types of vehicles are found significantly different. Detailed statistics are given in Table
1 and obtained speed distribution curves under free flowing condition for different vehicle types are shown below in Fig. 1.

Table 1

Detailed Statistics of the FFS from the Observed Data and Statistical Model

\begin{tabular}{|c|c|c|c|c|c|c|c|}
\hline \multirow[b]{2}{*}{ Vehicle type } & \multirow[b]{2}{*}{$\begin{array}{l}\text { Number of } \\
\text { observations }\end{array}$} & \multicolumn{3}{|c|}{ Statistics of observed data } & \multicolumn{3}{|c|}{ Statistics of fitted distribution* } \\
\hline & & $\begin{array}{l}\text { Mean } \\
(\mathrm{km} / \mathrm{h})\end{array}$ & $\begin{array}{l}\text { Standard } \\
\text { deviation } \\
(\mathrm{km} / \mathrm{h})\end{array}$ & C.v. & $\begin{array}{l}\text { Mean } \\
(\mathrm{km} / \mathrm{h})\end{array}$ & $\begin{array}{l}\text { Standard } \\
\text { deviation } \\
(\mathrm{km} / \mathrm{h})\end{array}$ & C.V. \\
\hline All & 720 & 55.73 & 13.70 & 0.25 & 55.72 & 13.72 & 0.25 \\
\hline Two wheeler & 164 & 49.96 & 11.60 & 0.23 & 50.33 & 11.54 & 0.23 \\
\hline Small car & 137 & 62.52 & 13.99 & 0.22 & 62.67 & 14.68 & 0.23 \\
\hline Big car & 148 & 62.59 & 13.53 & 0.22 & 62.48 & 14.12 & 0.23 \\
\hline Bus & 61 & 54.13 & 13.34 & 0.25 & 54.09 & 15.05 & 0.28 \\
\hline LCV & 122 & 49.42 & 9.19 & 0.19 & 48.77 & 9.57 & 0.20 \\
\hline Truck & 88 & 46.85 & 7.56 & 0.16 & 46.71 & 7.81 & 0.17 \\
\hline
\end{tabular}

*Normal Distribution; C.V. $=$ Coefficient of Variation

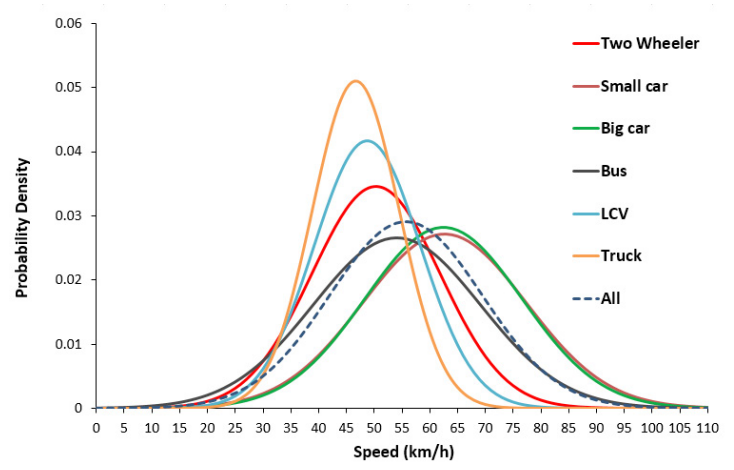

Fig. 1.

Speed Distribution Curves under Free Flowing Conditions for Different Vehicle Categories

Kolmogorov-Smirnov (K-S) test was performed to check the compatibility of the modelled distribution curves with the observed data. K-S test statistic was determined from the difference between the cumulative percentage of the observed and the expected frequency. Test statistic ' $D$ ' is the maximum value of these differences over the entire observed population interval and $a$ is the co-efficient of confidence level. P-value is the probability of obtaining a test statistic assuming that the null hypothesis is true.
Condition of acceptance of null hypothesis is as follows:

H0: $\quad \mathrm{P} \geq \alpha ; \quad$ Model fits to observed data

H1: $\quad \mathrm{P}<\alpha$; Model does not fit to observed data

Criterion: reject $\mathrm{HO}$ if $\mathrm{P}<\alpha$

Fitness test details for each type of vehicle is presented in Table 2 . 
Table 2

Goodness-of-Fit Test (K-S Test) Details of Normal Distribution Models

\begin{tabular}{lllll}
\hline Vehicle Type & Statistic 'D' & $\mathbf{P}$ - Value & $\boldsymbol{\alpha}$ - Value & $\begin{array}{l}\text { Null hypotheses } \\
\text { (Accept / Reject) }\end{array}$ \\
\hline All & 0.145 & 0.869 & 0.05 & Accept \\
Two wheeler & 0.208 & 0.470 & 0.05 & Accept \\
Small car & 0.223 & 0.629 & 0.05 & Accept \\
Big car & 0.165 & 0.847 & 0.05 & Accept \\
Bus & 0.273 & 0.280 & 0.05 & Accept \\
LCV & 0.219 & 0.410 & 0.05 & Accept \\
Truck & 0.178 & 0.494 & 0.05 & Accept \\
\hline
\end{tabular}

From the above Fig. 1, Table 1 and Table 2, following things are observed:

a) Speeds under free flowing conditions are normally distributed as all models for different vehicle categories are acceptable from the goodness-of-fit perspective. P-value for each type of vehicle is higher than the ' $\alpha$ ' value (0.05) for $95 \%$ confidence level.

b) A wide range of variation in FFS was noticed from 46.71 (for truck) $\mathrm{km} / \mathrm{h}$ to $55.72 \mathrm{~km} / \mathrm{h}$ (for small car) when FFS was considered category wise. It supports the above mentioned statement that different types of vehicle having different static and dynamic characteristics, would have different free-flow speeds.

c) It was observed that speed distribution curves of small car and big car under free-flowing condition almost coincide with each other. Also, FFS of small car and big car are nearly same and much greater than that of other types of vehicle.

d) Both LCV and truck have lower coefficient of variation value (below $0.2)$. Hence, it can be said that LCV and truck behave more consistently under low volume conditions but their freeflow speeds are below $50 \mathrm{~km} / \mathrm{h}$, very low compared to those of other vehicles.

\section{Sensibility of Percent Speed Reduction with Traffic Condition}

This phase of the study comprises of estimation of PSR and test of its sensibility with prevailing flow conditions. Category wise FFS values were taken from Table 1 and consequently, PSR was calculated for every individual vehicle. Average PSR was further determined coupled with corresponding v/c ranges as it is necessary to check how much this newly adopted performance measure is sensible to prevailing traffic condition. In Fig. 2, a second order polynomial relationship was found well fitted between PSR and v/c ratio which is globally considered as an effective measure for LOS assessment. A progressive growth of PSR is noticed with the increase of $\mathrm{v} / \mathrm{c}$ ratio. However, rate of increase in PSR drops gradually with increase in $\mathrm{v} / \mathrm{c}$ ratio. For low volume condition, a certain increase in flow results in an adequate rise in PSR. But gradually when flow approaches near the capacity, PSR does not rise much with a certain increase in $\mathrm{v} / \mathrm{c}$ ratio. Therefore from the above observations, 
it may be concluded that PSR is well sensible to traffic condition and can be considered as an effective performance measure for LOS assessment of an urban arterial.

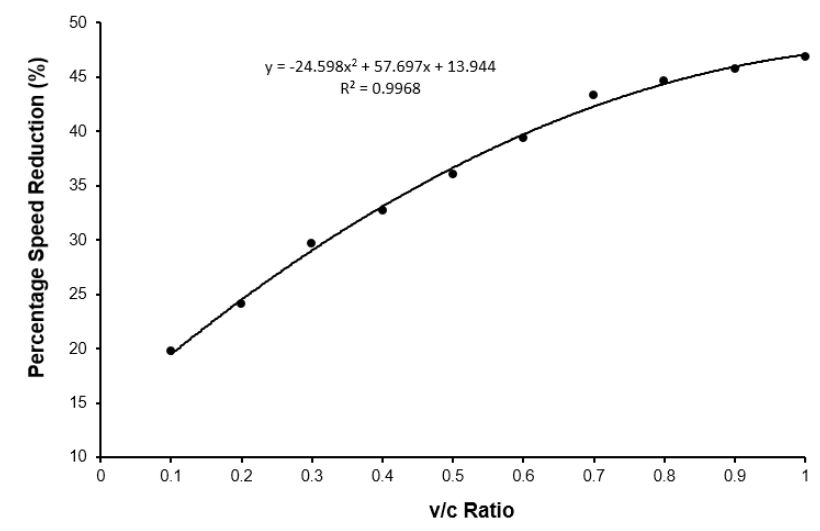

Fig. 2.

Change in Percentage Speed Reduction with the Change in V/C Ratio

\section{Assessment of LOS: A Clustering Approach}

K-mean clustering, one of the most popular clustering algorithms was employed to classify the collected PSR data in a number of subgroups. Each subgroup corresponds to one service level on urban arterial. Silhouette method was then adopted to validate the constructed clusters. PSR was primarily classified into six clusters as LOS A to LOS $\mathrm{F}$ are the six globally acknowledged service levels. Now if successive validation would become unsuccessful, plan of the study was to vary number of subgroups and results for each case were validated to find the optimum number of clusters.

\subsection{Cluster Analysis and Result}

Steps that were invariably followed in K-mean clustering process, are given below: Step 1: Number of clusters ' $k$ ' was decided firstly among which observed data would be divided.
Step 2: Observed data were randomly distributed among ' $k$ ' number of clusters. Step 3: Centre of each selected cluster was determined as the mean of the cluster members.

Step 4: Euclidean distances were measured between each data point and the centers of all clusters. This distance shows how close a data point is to the cluster center.

Step 5: If any observed data point was found to be closer to the center of any other class than its present one, that data point was then shifted to that cluster.

Step 6: Clusters including newly added data points were developed. This procedure was repeated from Step 3 successively until the maximum number of iteration was reached.

All the PSR values at wide range of flow scopes were taken together and the above exhibited procedure was repeated up to 100 iterations to obtain the range of each cluster. Threshold values of PSR for six LOS classes are shown in Fig. 3. 


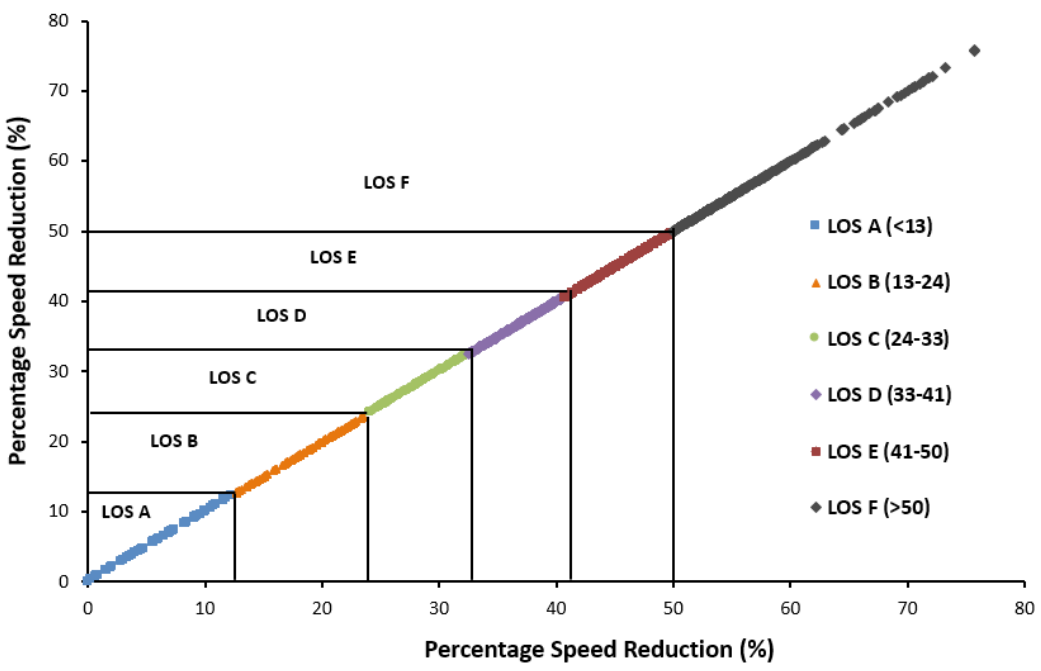

Fig. 3.

Six Clusters Signifying LOS Criteria Based on PSR

\subsection{Cluster Validation Measure: Application of Silhouette Method}

Cluster validation is to investigate the quality of clusters i.e. how good the cluster members are fitted within the range. For this purpose, Dunn's index and Silhouette index: two validity indices are commonly used in case when evaluation is done based on distance measure involving the observed data set themselves. No doubt, Dunn's index provides very accurate quality evaluation. But, major disadvantage of this index lies in its complex calculation which is very laborious and time consuming. Whereas only drawback of Silhouette index is that it cannot be used effectively for higher dimensional space which is not required in our present study. Therefore, Silhouette index was considered for validation measure of clusters.

Silhouette method which provides a graphical representation of how well each data point fits within a cluster, was introduced by
Rousseeuw (1987). Silhouette index for ith member is defined as per Eq. (2).

$S(i)=\frac{b(i)-a(i)}{\max \{a(i), b(i)\}}$

Where $a(i)$ is the average distance between $i$ th member and other members of the same cluster and $b(\mathrm{i})$ is the average distance between ith member and the members of nearest neighboring cluster. Hence, it is obvious that $-1 \leq \mathrm{S}(\mathrm{i}) \leq 1$. Generally, Silhouette value of each member under same cluster is displayed together and it forms a band. Height of the band simply shows the number of members in that cluster. Average of the Silhouette indices of all the members in a cluster is termed as Silhouette width of that specific cluster and the weighted average of all Silhouette widths for the complete data set denotes Silhouette co-efficient. Table 3 (Spector, 2011) displays ranges of Silhouette co-efficient and corresponding qualities of clustered data. 
Table 3

Silhouette Co-Efficient Criteria for Quality of Clusters

\begin{tabular}{ll}
\hline Range of Silhouette co-efficient & Interpretation \\
\hline $0.71-1.0$ & A strong structure has been found \\
$0.51-0.70$ & A reasonable structure has been found \\
$0.26-0.50$ & The structure is weak and could be artificial \\
$<0.25$ & No substantial structure has been found \\
\hline
\end{tabular}

Source: Spector (2011)

PSR data assigned to different classes, were taken into consideration for validity checking using Silhouette Technique. Silhouette index for each member was determined using Eq. (3). Consequently Silhouette width for each cluster and Silhouette co-efficient were computed taking average of Silhouette indices and Silhouette widths respectively. Silhouette co-efficient was found to be 0.572 (Fig. 4). Hence as per Table 3, it can be said that a reasonable structure was found and developed clusters were proved to be compatible with the observed PSR data.

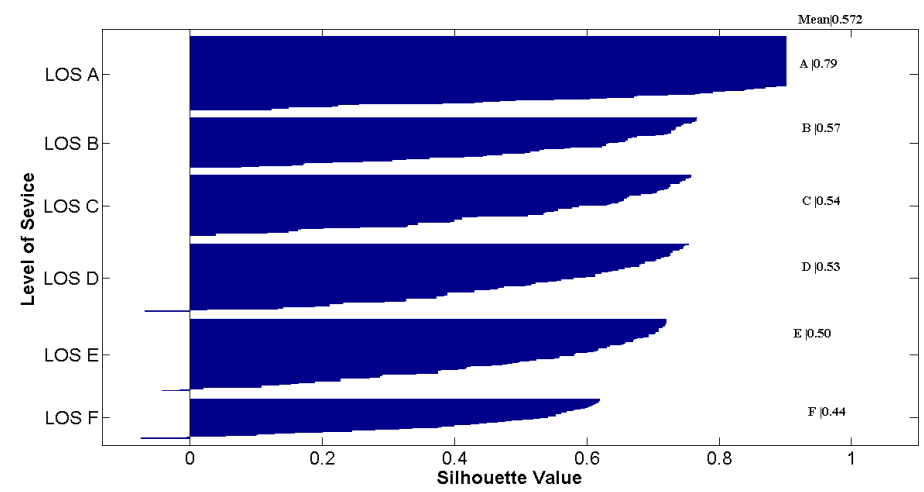

Fig. 4.

Silhouette Plot of K-Mean Clustering Showing Silhouette Widths

Six LOS were therefore proposed along with relevant PSR ranges (Table 4). A vehicle enjoys LOS A on urban arterial moving at speed $13 \%$ or less reduced from FFS of that type of vehicle. Similarly, up to $24 \%, 33 \%, 41 \%$ and $50 \%$ percent speed reduction ensures LOS B, LOS C, LOS $\mathrm{D}$ and LOS E respectively. Finally when PSR exceeds 50 or in other words average travel speed drops below half of the freeflow speed, the worst service level LOS $\mathrm{F}$ appears. 
Table 4

PSR Ranges for Different LOS of Urban Road under Mixed Traffic Condition

\begin{tabular}{ll}
\hline Level of service & Percentage Speed Reduction (PSR) \\
\hline LOS A & $\leq 13$ \\
LOS B & $>13-24$ \\
LOS C & $>24-33$ \\
LOS D & $>33-41$ \\
LOS E & $>41-50$ \\
LOS F & $>50$ \\
\hline
\end{tabular}

\section{Conclusions}

In the present study, a methodology to evaluate LOS of urban arterial was proposed. To this end, using the same methodology LOS criteria (Table 4) were proposed based on the field data collected from a six lane divided urban arterial in Kolkata. Apart from this contribution, some major findings of this study are summarized below:

i) Free flow speeds of different vehicle categories were determined (Table 1) and it was observed that FFS varies largely depending upon the types of vehicle. Small sized vehicles (except two wheeler) have higher free-flow speed than the larger ones. Result had shown that LCV and truck behave more consistently during low volume condition compared to other types of vehicles.

ii) Percentage Speed Reduction (PSR) from FFS was identified as a performance measure for LOS assessment on urban arterials. Increase in PSR signifies the decrease in service quality of the road. Also, it was observed that PSR increases with the increase in $\mathrm{v} / \mathrm{c}$ ratio and corresponding relationship was formulated. iii) Silhouette method found six clusters formed by K-mean clustering technique, 'a reasonable structure' having good compatibility with PSR data. In PSR based LOS criteria, it was observed that operating condition falls into worst level i.e. LOS F when Percentage Speed Reduction appears greater than $50 \%$.

To culminate in the discussion, it can be said that the methodology suggested in this study could be useful for evaluating LOS criteria of urban arterial in any other contexts and conditions. Also as a further scope of the study, it can be extended to undivided urban roads where the extent of conflict with opposite directional traffic would influence level of service on the road.

\section{References}

Bhuyan, P.K.; Rao, K.V.K. 2011. Defining level of service criteria of urban streets in Indian context, European Transport, 49(17): 38-52.

Das, A.K.; Patnaik, A.K.; Dehury, A.N.; Bhuyan, P.K.; Chattaraj, U.; Panda, M. 2013. Defining level of service criteria of urban streets using Neural Gas Clustering, IOSR Journal of Engineering, 3(5): 18-25. 
Lal, A.K.; Clement, R.W. 2005. Economic development in India: The role of individual enterprise (and entrepreneurial spirit), Asia Pacific Development Journal, 12(2): 81-99.

Maitra, B.; Sikdar, P.K.; Dhingra, S.L. 1999. Modeling congestion on urban roads and assessing level of service, Journal of Transportation Engineering, 125(6): 508-514.

Marwah, B.R.; Singh, B. 2000. Level of service classification for urban heterogeneous traffic: A case study of Kanapur metropolis, Transportation Research Circular, E-C018: 271-286.

Rao, A.M.; Rao, K.R. 2012. Measuring urban traffic congestion - A review, International Journal for Traffic and Transport Engineering, 2(4): 286-305.

Rousseeuw, P.J. 1987. Silhouettes: A graphical aid to the interpretation and validation of cluster analysis, Journal of Computational and Applied Mathematics, 20: 53-65.
Singh, J. 2014. India's automobile industry: Growth and export potential, Journal of Applied Economics and Business Research, 4(4): 246-262.

Spector, P. 2011. Stat 133 Class Notes. University of California, Barkeley. Available from Internet: <http:// www.stat.berkeley.edu/ s133/all2011.pdf $>$.

Transportation Research Board. 1965. Highway Capacity Manual. Washington D.C.

Transportation Research Board. 1985. Highway Capacity Manual. Washington D.C.

Transportation Research Board. 2000. Highway Capacity Manual. Washington D.C.

Transportation Research Board. 2010. Highway Capacity Manual. Washington D.C. 